\title{
Switching in coupled nonlinear photonic-crystal resonators
}

\author{
Björn Maes, Peter Bienstman, and Roel Baets \\ Department of Information Technology, Ghent University—Interuniversity Micro Electronics Center, St.- \\ Pietersnieuwstraat 41, 9000 Ghent, Belgium
}

\begin{abstract}
Received November 18, 2004; revised manuscript received February 23, 2005; accepted February 28, 2005
Using coupled-mode theory we examine the linear and Kerr nonlinear behavior of multiple consecutive photonic-crystal switches. Two types of resonators are considered, those with the cavity inside and those adjacent to the waveguide. We observe gap solitons in both structures and examine a nonlinear mode with energy localized near the boundaries of the finite system. Finally, we propose a device with two side-coupled resonators and a judiciously chosen intercavity distance that demonstrates switching at low powers. In addition to coupled-mode theory, rigorous simulations are performed for this structure. (C) 2005 Optical Society of America OCIS codes: $190.3270,190.5530,230.4320$.
\end{abstract}

\section{INTRODUCTION}

Systems with waveguides and resonators are nowadays widely studied because of their ability to tune coupling strengths and resonance frequencies for many purposes. If chains of these devices are examined, new effects and switching opportunities arise. ${ }^{1-3}$ This has been examined for many types of structures, including distributedfeedback mirrors, ring resonators, and photonic crystal (PC) defects. ${ }^{4,5}$ The PC structures have the potential to provide ultracompact photonic integrated structures of only a few micrometers. ${ }^{6,7}$ Because of the bandgap, all the light in two-dimensional (2D) PC structures remains confined in the waveguides, therefore analysis of the devices becomes easier and radiation losses can be ignored. Moreover, the strong confinement makes possible the use of weak nonlinear effects.

In this article we study the linear and nonlinear continuous-wave behavior of chains of $\mathrm{PC}$ resonator devices using coupled-mode theory (CMT) ${ }^{8}$ This theory has been used successfully for many geometries. ${ }^{9}$ In Ref. 10 CMT was extended to handle Kerr nonlinear resonators. Here we use these equations in a transfer-matrix scheme to calculate sequences consisting of two kinds of cavities: The resonant coupling and the side coupling case, as shown in Figs. 1(a) and 1(b), respectively. In the resonantcoupling structure the resonator blocks the waveguides; therefore light is transmitted only on resonance. In contrast, in the side-coupling case there is a high reflection on resonance. ${ }^{11}$ We focus on finite sequences with identical resonators. However, the formalism can also be used for chains with nonuniform parameters, e.g., apodized structures.

If series are examined, the relation with Bloch modes in infinite structures can be considered. Where light is strongly reflected, gaps appear. The resonant-coupled case gives rise to a transmission band, whereas the sidecoupled cavities can amount to a resonator gap. However, owing to nonlinearity a finite system can switch from a highly reflective state in the gap to a state with unity transmission. This state is called the gap soliton. ${ }^{12-16}$ These nonlinear states, also considered as intrinsic localized modes, have consequences for energy localization and appear in different physical settings. ${ }^{17}$ We demonstrate gap solitons in both the resonant-coupled and sidecoupled geometry. Furthermore, nonlinear transmission resonances without a linear origin can be examined. ${ }^{17} \mathrm{Fi}$ nally, we show a new kind of nonlinear resonance with an alternative energy profile, where the energy is localized on the boundaries of the finite series.

An important parameter for these coherently interacting cavities is the length of the intermediate waveguide, which can be tuned to change the phase and interference characteristics. The linear spectrum of two side-coupled resonators can exhibit a very narrow transmission peak. ${ }^{4}$ We shall exploit this peak in an actual design, to demonstrate efficient switching both with CMT and more exact simulations. ${ }^{18-22}$

In Section 2 we describe the CMT calculation methods. We use this for a description of the linear spectra for both geometries in Section 3. Studies of different types of nonlinear resonances and gap solitons are performed in Section 4. Finally, in Section 5 we present the concrete PC device consisting of two side-coupled resonators.

\section{CALCULATION METHOD}

\section{A. Resonant Coupling}

Throughout this paper we assume that the waveguides and resonators are single mode in the frequency region of interest. Furthermore, the resonators are placed sufficiently far from each other that interaction occurs only via the intermediate waveguides. Then, with CMT, the fields in a single cell of the resonant coupling geometry [see Fig. 1(a)] are described by ${ }^{8}$

$$
\frac{\mathrm{d} a}{\mathrm{~d} t}=\left[j\left(\omega_{r}+\delta \omega\right)-\frac{1}{\tau}\right] a+d f_{1}+d b_{2},
$$




$$
\begin{aligned}
& b_{1}=\exp (j \phi) f_{1}+d a, \\
& f_{2}=\exp (j \phi) b_{2}+d a .
\end{aligned}
$$

Here $a$ denotes the complex cavity-mode amplitude, whereas $f_{1}$ and $f_{2}\left(b_{1}\right.$ and $\left.b_{2}\right)$ denote the forward (backward) waveguide Bloch-mode amplitudes. The cavity lifetime is $\tau$ and the resonance frequency is $\omega_{r}$, with $Q$ $=\tau \omega_{r} / 2$. In the previous $d=j \exp (j \phi / 2) / \sqrt{\tau}$, where the phase $\phi$ depends on the waveguide and the PC reflection properties. $\delta \omega$ indicates the nonlinear resonance frequency shift:

$$
\delta \omega=-\frac{|a|^{2}}{P_{0} \tau^{2}},
$$

where $P_{0}$ is the characteristic nonlinear power of the cavity. ${ }^{10,23}$ The amplitudes are normalized so that $|a|^{2}$ is equal to the energy in the resonator and $\left|f_{i}\right|^{2}\left(\left|b_{i}\right|^{2}\right)$ indicates the power in the forward (backward) mode of waveguide $i$. The previous equations are valid for resonator modes with a vertical mirror plane through the center, e.g., monopoles. These equations are adjusted for modes with a vertical nodal plane, e.g., horizontal dipoles, by changing the sign of the last term in Eqs. (1) and (3). Here we will work in the cw regime, so $\mathrm{d} / \mathrm{d} t$ is simply replaced by $j \omega$ in Eq. (1). In this regime we can analyze the possible states of the system. Specific excitations to reach or avoid the states need to be examined in the time domain. $^{11}$

In the resonant-coupled geometry it is easy to concatenate cells with a transfer-matrix approach. We work from right to left, after fixing the output power. With Eq. (3) we determine $a$, which we then use in Eq. (1) to obtain $f_{1}$. Finally, with Eq. (2) we get $b_{1}$. In this way we calculate noniteratively through a chain of nonlinear resonators. Note that both the stable and unstable states can be determined.

\section{B. Side Coupling}

In the side-coupling geometry [see Fig. 1(b)] an analogous set of equations is derived:

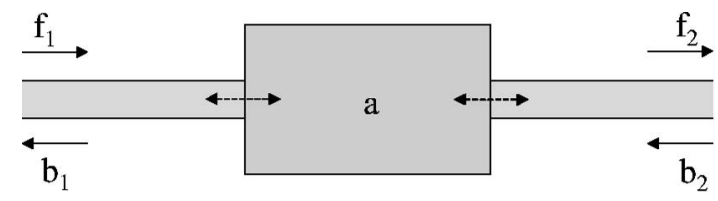

(a)

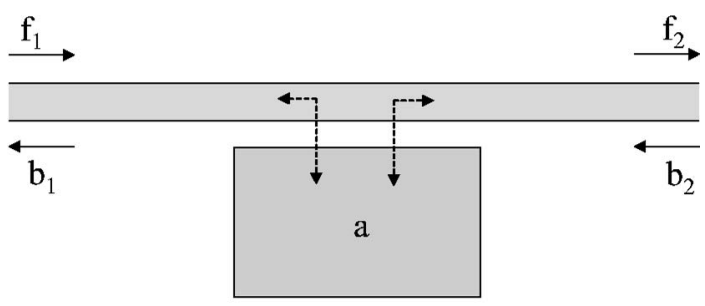

(b)

Fig. 1. Two geometries with resonator and waveguide amplitudes. (a) Resonant-coupled structure, (b) Side-coupled structure.

$$
\begin{aligned}
\frac{\mathrm{d} a}{\mathrm{~d} t} & =\left[j\left(\omega_{r}+\delta \omega\right)-\frac{1}{\tau}\right] a+d f_{1}+d b_{2}, \\
f_{2} & =\exp (j \phi) f_{1}+d a \\
b_{1} & =\exp (j \phi) b_{2}+d a
\end{aligned}
$$

Again $d=j \exp (j \phi / 2) / \sqrt{\tau}$, but here $\phi=-k_{z} n p$, with $k_{z}$ as the waveguide-mode propagation constant, $n$ as the number of periods and $p$ as the PC period. Note that these equations are equivalent to a model of a ring resonator adjacent to two waveguides. ${ }^{24}$

A direct transfer-matrix approach is possible here only for linear resonators. Calculating a nonlinear series has to be done differently. With Eqs. (5) and (6) we obtain a nonlinear equation with $f_{2}, b_{2}$, and $a$. With the fixed $f_{2}$ and $b_{2}$ and starting from a guess resonator amplitude $a$, we can iteratively solve this equation with, e.g., NewtonRaphson. Finally, using Eqs. (6) and (7) we obtain the fields $f_{1}$ and $b_{1}$ on the left side of the cell.

\section{LINEAR TRANSMISSION}

In this section we give an overview of the linear transmission spectra for both geometries. Here, the two main parameters are the number of resonators $N$ and the phase $\phi$. We can fix this phase to a desired value by adjusting the number of waveguide periods in a cell, as the spectra are $2 \pi$ periodic in $\phi$. Of course, the cw calculations will accurately represent short-pulse excitations only if the structure is sufficiently short. Furthermore, because of fabrication and loss issues, actual designs need a minimum number of periods.

The spectra in this section ignore dispersion. For real PC structures the main effect is waveguide dispersion. However, this $k_{z}$ dependence on $\omega$ can be included. Moreover, with high- $Q$ resonators it is sufficient to use a constant $k_{z}$ or a linear $k_{z}(\omega)$ function. Without dispersion the spectra are only symmetric around $\omega_{r}$, if $\phi=n \pi$ or $\phi$ $=\pi / 2+n \pi$ (where $n$ is an integer). These values represent the special cases. Spectra calculated in this section are plotted versus $\delta=\tau\left(\omega-\omega_{r}\right)$.

\section{A. Resonant Coupling}

Now we discuss the resonant-coupling case in more detail. In general the transmission spectrum consists of $N$ peaks that shift in function of $\phi$. Only the central peak at $\omega_{r}$ remains stationary. If $\phi=\pi / 2$ these peaks are closest together, as shown in Fig. 2. For even values of $N$ the central two peaks coalesce into one broader peak. As $\phi$ approaches 0 the $N-1$ nonstationary peaks move (infinitely) farther away from $\omega_{r}$. In the case of $N=2$, e.g., one can calculate that the position of the moving peak varies as $\delta_{\text {peak }}=-\cot \phi$. When $\phi=0$ all resonators have the same magnitude and the same (or opposite) phase; they behave as one cavity. For this case several spectra are shown in Fig. 3. They are simply proportional to the spectrum of one resonator, and have a width $1 /(N \tau)$.

When $N$ increases the situation becomes comparable with infinite structures, and we can use Bloch modes. These are studied via 


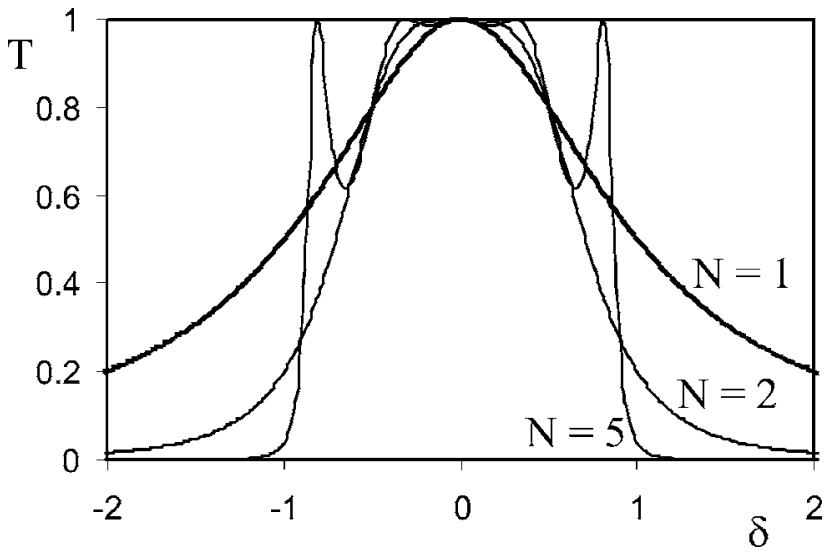

Fig. 2. Transmission spectra for resonant coupled geometry with $\phi=\pi / 2$ and $N=1,2$, and 5 .

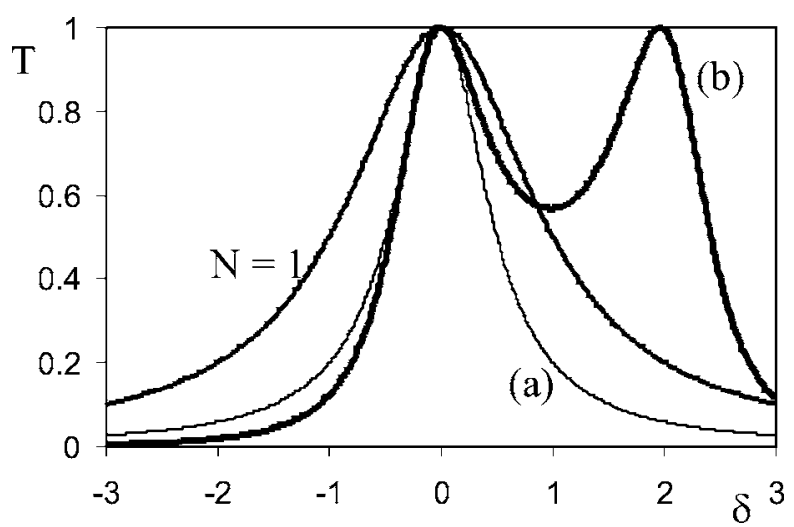

Fig. 3. Transmission for resonant coupling. $N=1$ is indicated, whereas curves (a) and (b) correspond to $N=2$ but with $\phi=0$ and $\phi=-0.15 \pi$, respectively.

$$
\left[\begin{array}{l}
f_{2} \\
b_{2}
\end{array}\right]=\exp (j \beta \Lambda)\left[\begin{array}{l}
f_{1} \\
b_{1}
\end{array}\right]=\mathbf{T} \cdot\left[\begin{array}{l}
f_{1} \\
b_{1}
\end{array}\right],
$$

where $\beta$ is the Bloch-mode propagation constant and $\Lambda$ is the length of a unit cell. This cell must not be confused with a period of the waveguide but corresponds to an entire element of the chain as depicted in Fig. 1(a). The transfer matrix $\mathbf{T}$ is obtained from Eqs. (1)-(3). In a bandgap, the amplitude of its eigenvalues are smaller than 1 or, equivalently, $\beta$ has a nonzero imaginary part. The resulting diagram with the resonator transmission regions is shown in Fig. 4 and agrees with the finite results of, e.g., $N=5$ in Fig. 2. The center separating curve corresponds to $\tan (\phi / 2)$ The case $\phi=0$ is again highlighted as a special value.

\section{B. Side Coupling}

There are many similarities between the side-coupling and resonant-coupling structures. Indeed, for a singleresonator reflection and transmission are exchanged, immediately exposing the duality. Thus the resonator transmission band of the previous section becomes a resonator reflection band or resonator gap. However, specific features will become apparent. ${ }^{4}$ Here, the transmission spectrum of $N$ resonators has $N-1$ peaks with unity transmission, see Fig. 5. For $\phi=\pi / 2$ these peaks are evenly distributed, one half on the lower edge, the other half on the upper edge. For even numbers one peak moves to infinity at this phase. If $\phi$ decreases from $\pi / 2$ to 0 , the maximum peaks first shift to one side of $\omega_{r}$ then gradually disappear at $\omega_{r}$. Before dissolving, these peaks become extremely small, as shown in Figs. 6 and 7. At $\phi=0$ the spectra are proportional to the dip for $N=1$ with width $N / \tau$. We will exploit the narrow resonances with small $\phi$ for nonlinear switching in Section 5 . Here, we give a simple description of this peak for two resonators. The transmission is calculated for $N=2$ from Eqs. (5)-(7):

$$
T=\frac{\delta^{4} /\left(4 \cos ^{2} \phi\right)}{\delta^{4} /\left(4 \cos ^{2} \phi\right)+(\delta-\tan \phi)^{2}} .
$$

For small $\delta \ll 1$ and $\phi \ll 1$ this describes a Lorentzian peak around $\tan \phi$ with width proportional to $\delta^{4}$. These maxima are Fabry-Perot resonances that also appear in nonuniform grating structures. ${ }^{25}$

Using the Bloch relation Eq. (8) but with the transfer matrix from Eqs. (5)-(7) we can determine the resonator gaps in function of $\phi$. The transmission band of the previous section directly corresponds to the resonator gap here, so we can use the same diagram of Fig. 4.

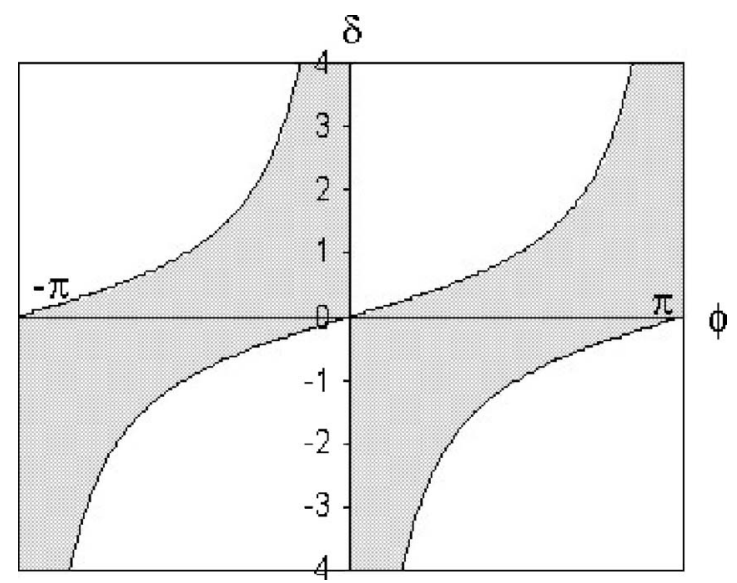

Fig. 4. Bloch mode results. For the resonant-coupled structure the shaded region indicates the transmission band; for the sidecoupled geometry it represents the resonator gap.

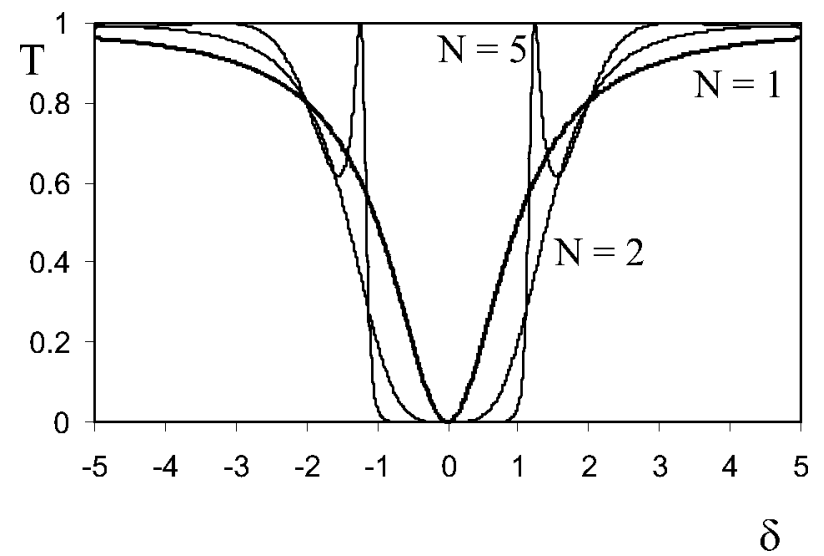

Fig. 5. Transmission spectra for side-coupled geometry with $\phi=\pi / 2$ and $N=1,2$, and 5 . 


\section{GAP SOLITONS AND NONLINEAR RESONANCES}

Generally speaking, when there is a frequency gap of some origin, light is exponentially dampened in the structure. However, in nonlinear media when the field has the required strength and shape, it is able to propagate through the gap with complete transmission. Such field excitations are called gap solitons, and they appear in both finite and infinite settings. Chen and Mills ${ }^{12}$ were the first to theoretically report gap solitons in finite distributed Bragg reflectors, but these solitons appear in nu-

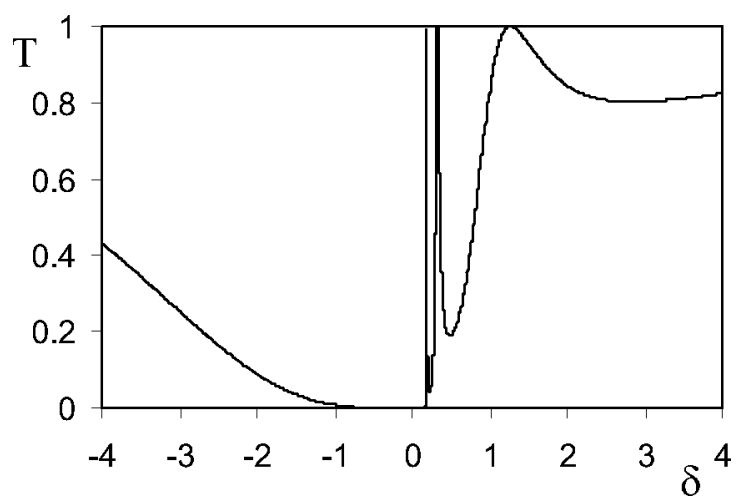

Fig. 6. Transmission for side coupling with $\phi=0.1 \pi$ and $N=4$.

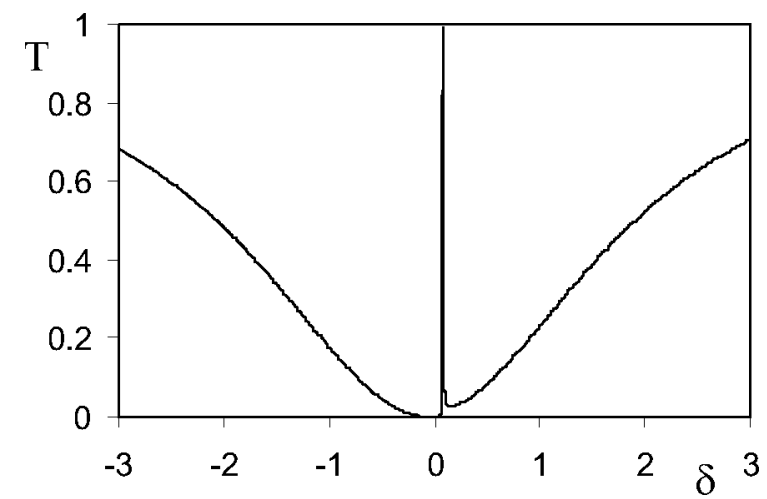

Fig. 7. Transmission for side coupling with $\phi=0.025 \pi$ and $N=2$.

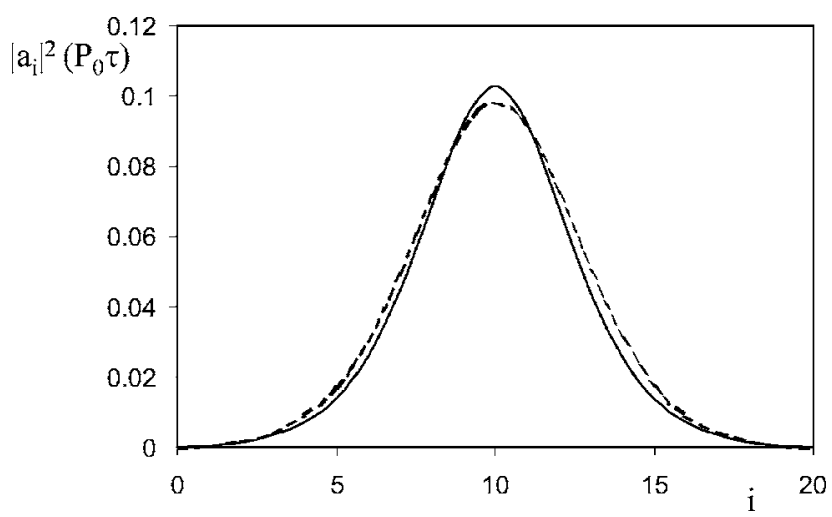

Fig. 8. Fundamental gap solitons for resonant-coupling (solid curve) and side-coupling (dashed curve) geometry, with necessary input powers $0.94 \times 10^{-4} P_{0}$ and $1.08 \times 10^{-4} P_{0}$, respectively. $a_{i}$ is the amplitude of resonator number $i$. The energy profiles follow a square hyperbolic secant curve.

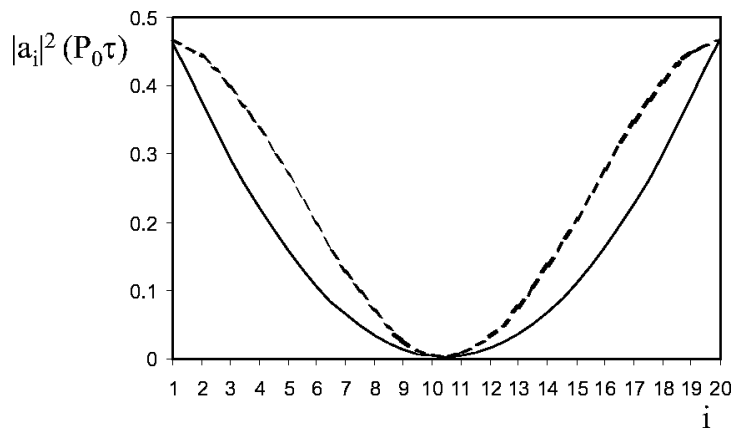

Fig. 9. Resonator energy profile of the nonlinear switching mode for 20 side-coupled cavities. The dashed curve shows the corresponding linear resonance profile at $\delta=-1.3$, normalized according to the maximum nonlinear value. The solid curve shows the nonlinearly adjusted resonance with $\delta=-1.6$ and input power $0.6 P_{0}$.

merous other systems. ${ }^{13-16}$ Here, we find the same type of fundamental gap solitons in both geometries.

In Fig. 8 we show the hyperbolic secant profiles of the resonator amplitudes for $N=21$. For the calculations in this section we use $Q=1000, P_{0}=0.5 \times 10^{5} \mathrm{~W} / \mathrm{m}, \omega_{r}=0.4$ $\times(2 \pi c / p)$, and $p=0.6 \mu \mathrm{m}$. We choose $\delta=-1.05$ for the resonant-coupled case and $\delta=0.95$ for the side-coupled case. These frequencies are near the upper frequency edge of the gap with $\phi=\pi / 2$, so in combination with positive nonlinearity, gap soliton switching is possible. The higher-order, multiple-hump modes can also be observed in these systems.

The previous modes develop from linear transmission maxima. Small detuning from the linear peak gives a profile of the nonlinear mode that is similar to the linear profile. By increasing the detuning the nonlinear mode evolves into the hyperbolic secant shape, because the center maximum acts as a nonlinear energy trap for the field. The same idea can be applied to find other types of nonlinear modes. Therefore we turn our attention to the peaks in the spectrum of side-coupled resonators near $\phi$ $=0$, see Fig. 6 . The rightmost peak in this spectrum has a profile with a minimum in the center and two maxima on the input and output boundaries. This is different from the gap solitons, and indeed allows for energy localization near the interfaces. In order to have reasonable contrast switching we choose $\phi$ to locate the peak near the left side of $\omega_{r}$. This is possible, as it is the last peak to disappear as $\phi$ approaches zero from the negative side. With $\phi=$ $-0.005 \pi$ and $N=20$, a linear maximum is located at $\delta=$ -1.3. We switch from $\delta=-1.6$ with transmission $T=0.34$ toward this state. The nonlinear mode profile, together with the normalized original linear profile, is shown in Fig. 9. Clearly the nonlinearity adjusts the mode, creating a different profile with energy near the edges.

In contrast, resonances from a purely nonlinear origin are also possible. ${ }^{17}$ In Fig. 10 we show the transmission versus $\delta$ for a certain fixed input power in a resonantly coupled system of five resonators. The same parameters as above are used, so $Q=1000, P_{0}=0.5 \times 10^{5} \mathrm{~W} / \mathrm{m}, \omega_{r}$ $=0.4 \times(2 \pi c / p)$, and $p=0.6 \mu \mathrm{m}$. We choose $\phi=\pi / 2$ in order to have a large bandgap region. The branch with maximum transmission near (a) has no linear origin. It actually consists of two nearby curves, and the one with high- 


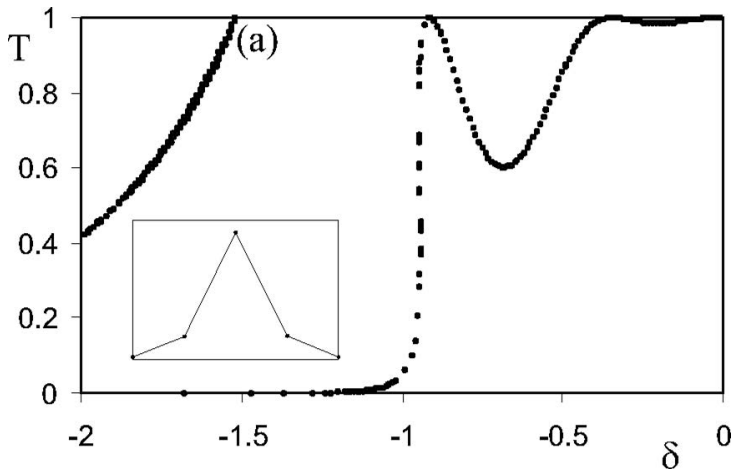

Fig. 10. Transmission versus $\delta$ for a resonant coupled system with $N=5$ at fixed input power $0.02 P_{0}$. The gap soliton originating from a nonlinear resonance is indicated by (a), and the energy profile $\left|a_{i}\right|^{2}$ is shown in the inset.

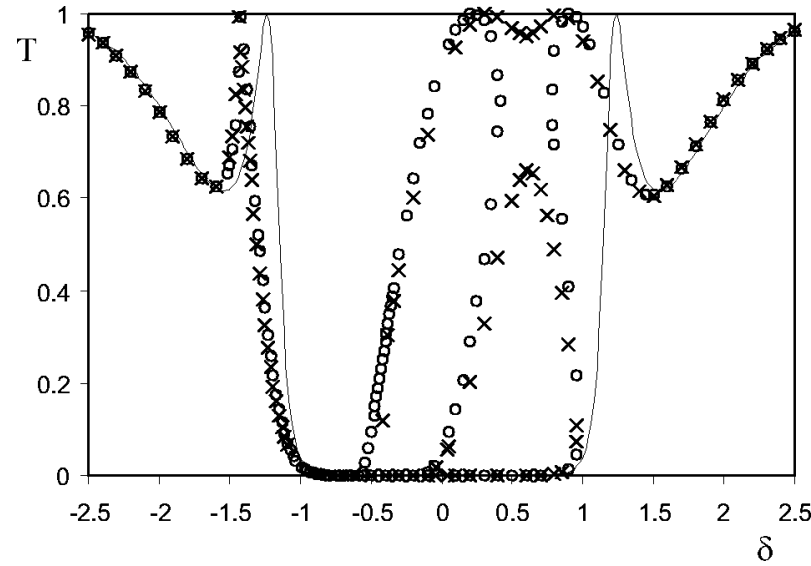

Fig. 11. Transmission versus $\delta$ for a side-coupled system with $N=5$ at fixed-input powers $P_{1}=0.08 P_{0}$ (circles) and $P_{2}=0.09 P_{0}$ (crosses). The linear transmission is also shown with a solid curve.

est transmission is stable. The inset in Fig. 10 shows the energy profile of the gap soliton state indicated by (a) in the figure. Note that by increasing the input power, the maximum (a) moves to the right. Eventually it reaches and interacts with the gap soliton of linear origin. Transmission then drops below one, so these soliton states no longer exist above this power threshold.

We have also found these nonlinear transmissions in side-coupled systems; they show the same behavior as in the other geometry. This is presented in Fig. 11, where we use the same parameters. The transmission with two different input powers and the linear curve is plotted. The purely nonlinear states in the gap are evident. The interaction with the gap soliton of linear origin is visible at input power $P_{2}$.

In this section we gave an overview of possible nonlinear states in both systems. We will now turn our attention to a more concrete switching example.

\section{SWITCHING}

Here we focus on two side-coupled resonators and look at the switching opportunity of the narrow peak appearing with small $\phi$; see Fig. 7 . We design a PC structure and calculate the linear and nonlinear characteristics with rigorous simulations. For this purpose we use the modeexpansion method and a nonlinear extension. ${ }^{18-22}$

The structure is shown in Fig. 12. The PC is a square lattice with period $p=0.6 \mu \mathrm{m}$ of square rods with diameter $d=0.4 p$. The rods have index 3.5 in an air background. A waveguide is formed by removing a row of rods. The two larger rectangles create the resonators and have sides $p$ and $0.48 p$. We use TM polarization, thus with one electric field component parallel to the rods. The linear transmission for one and two resonators is plotted in Fig. 13, both with CMT and with mode expansion. From the single-resonator transmission we get $\omega_{r}=0.3668767$ $\times(2 \pi c / p)$ and $\tau=19475.8 /(2 \pi c / p)$. Thus the resonator has $Q=\omega_{r} \tau / 2=3573$. In the region around $\omega_{r}$ the waveguide phase is $-k_{z} p=-1.6731$. With a cell of thirteen periods, and thus twelve periods between the resonators, the phase equals $\phi=0.2408$. The CMT curve with these parameters agrees well with the exact simulations.

For the nonlinear CMT calculations we need the characteristic power $P_{0}$ of the resonator. This power can be determined from an integral or a fitting to a nonlinear input-output curve from rigorous simulations. ${ }^{11}$ We used the latter approach and obtained $P_{0}=0.32086 \times 10^{4} \mathrm{~W} / \mathrm{m}$. Here the resonator rectangle is considered nonlinear, with Kerr coefficient $n_{2}=2 \times 10^{-19} \mathrm{~m}^{2} / \mathrm{V}^{2}$. The nonlinear index change is $n_{2}|\mathbf{E}|^{2}$, with $\mathbf{E}$ as the electric field.

Then we performed nonlinear exact simulations with two resonators separated by twelve periods. We choose $\omega=0.366883(2 \pi c / p)$, which is next to the transmission maximum. The bistable transmission curve is shown in Fig. 14 and agrees with the CMT calculations. It is clear from the scale in the figure that lower powers are required for switching than with one resonator. Of course, two resonators have twice the amount of nonlinear material, but the main reason is the narrow maximum. Although this peak is only approximately Lorentzian, its fitted $Q$ is $\sim 40$ times larger than the $Q$ of one resonator. Because $P_{0}$ is proportional to the inverse square of $Q$, we can estimate the characteristic power of our device to be 3 orders of magnitude smaller and thus more power efficient. The previous argument is more concrete if we compare the input power at the bistability threshold of one and two resonators. However, this comparison is not strict, because the systems have different nonlinear behavior. For one resonator bistability appears if $\delta<-\sqrt{3}$, so we choose $\delta=-\sqrt{3}$ and obtain at the switching point (where the transmission decreases rapidly) $P_{\text {in }}=1.347 P_{0}$. For the device with two resonators the bistability threshold is $\omega=0.3668863 \times(2 \pi c / p)$ and at the switching point

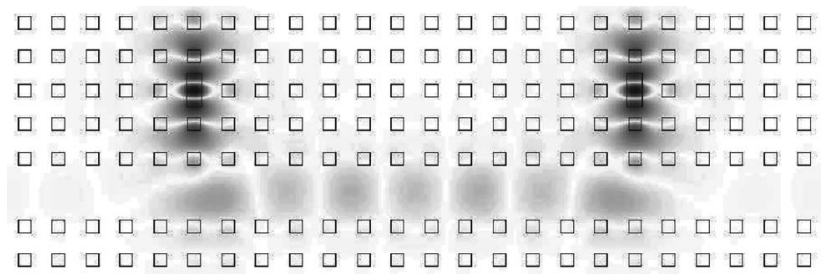

Fig. 12. Structure of the PC switching device. The electric field at the transmission maximum is superimposed. Because of high $Q$ the waveguide field is very small compared with the resonator fields. 


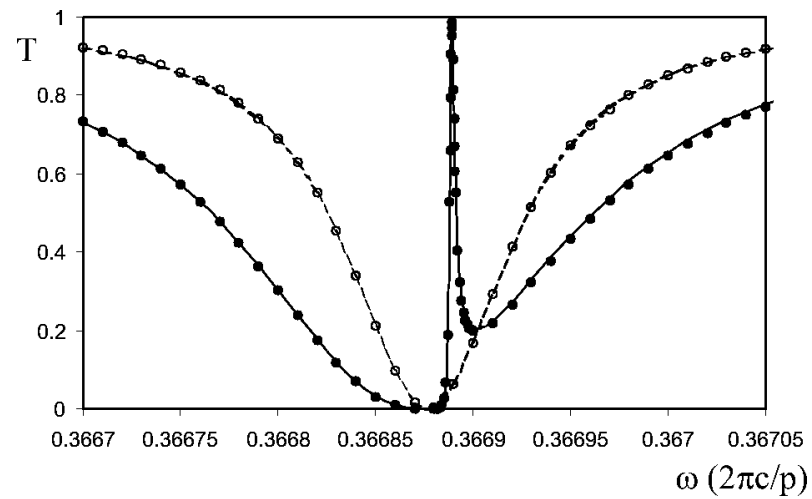

Fig. 13. Linear transmission for one and two resonators. The dashed (solid) curve represents the CMT calculations, whereas the circles (dots) show the rigorous simulations for one (two) resonator(s), respectively.

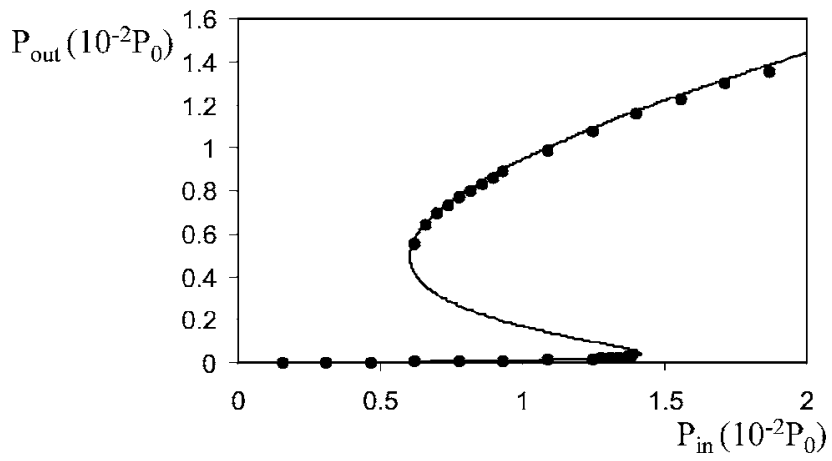

Fig. 14. Output power versus input power for the two-resonator device at $\omega=0.366883(2 \pi c / p)$. The CMT calculation is shown with a solid curve, the dots represent rigorous simulations.

(where transmission increases rapidly) $P_{\text {in }}=2.52$ $\times 10^{-3} P_{0}$. This corresponds indeed with a factor $10^{3}$.

\section{CONCLUSION}

The CMT is a flexible tool for study of a large variety of linear and nonlinear devices. In further work, one can use the framework defined above to include, e.g., apodization or reflecting waveguide sections with Fano-type transfer functions. ${ }^{8}$ Here, we examined series of resonators inside or adjacent to the waveguide. In both systems gap solitons were observed. In addition we found solitons originating from a nonlinear resonance. In the side-coupled geometry we observed a new nonlinear mode with energy located at the boundaries. With a correctly chosen interresonator distance, a narrow linear transmission peak in the spectrum of two side-coupled resonators appears. We designed a PC device to exploit the nonlinear switching near this peak and demonstrated its efficient operation with both CMT and rigorous calculations.

\section{ACKNOWLEDGMENTS}

Björn Maes and Peter Bienstman acknowledge the Flemish Fund for Scientific Research (FWO-Vlaanderen) for a doctoral and postdoctoral fellowship, respectively. Parts of this work were performed in the context of the Belgian DWTC project IAP-Photon.
Björn Maes can be reached by e-mail at bjorn.maes@intec.ugent.be.

\section{REFERENCES}

1. S. D. Gupta and G. S. Agarwal, "Dispersive bistability in coupled nonlinear Fabry-Perot resonators," J. Opt. Soc. Am. B 4, 691-695 (1987)

2. H. A. Haus and Y. Lai, "Theory of cascaded quarter wave shifted distributed feedback resonators," IEEE J. Quantum Electron. 28, 205-213 (1992).

3. A. Yariv, Y. Xu, R. K. Lee, and A. Scherer, "Coupledresonator optical waveguide: a proposal and analysis," Opt. Lett. 24, 711-713 (1999).

4. Y. Xu, Y. Li, R. K. Lee, and A. Yariv, "Scattering-theory analysis of waveguide-resonator coupling," Phys. Rev. E 62, 7389-7404 (2000).

5. J. E. Heebner, R. W. Boyd, and Q. H. Park, "SCISSOR solitons and other novel propagation effects in microresonator-modified waveguides," J. Opt. Soc. Am. B 19, 722-731 (2002).

6. W. Bogaerts, D. Taillaert, B. Luyssaert, P. Dumon, J. Van Campenhout, P. Bienstman, D. Van Thourhout, R. Baets, V. Wiaux, and S. Beckx, "Basic structures for photonic integrated circuits in silicon-on-insulator," Opt. Express 12, 1583-1591 (2004)

7. P. Sanchis, J. Garcia, J. Marti, W. Bogaerts, P. Dumon, D. Taillaert, R. Baets, V. Wiaux, J. Wouters, and S. Beckx, "Experimental demonstration of high coupling efficiency between wide ridge waveguides and single-mode photonic crystal waveguides," IEEE Photonics Technol. Lett. 16, 2272-2274 (2004).

8. S. Fan, W. Suh, and J. D. Joannopoulos, "Temporal coupledmode theory for the Fano resonance in optical resonators," J. Opt. Soc. Am. A 20, 569-572 (2003).

9. C. Manolatou, M. J. Khan, S. Fan, P. R. Villeneuve, H. A. Haus, and J. D. Joannopoulos, "Coupling of modes analysis of resonant channel add-drop filters," IEEE J. Quantum Electron. 35, 1322-1331 (1999).

10. M. Soljacic, M. Ibanescu, S. G. Johnson, Y. Fink, and J. D. Joannopoulos, "Optimal bistable switching in nonlinear photonic crystals," Phys. Rev. E 66, 055601 (2002).

11. M. F. Yanik, S. Fan, and M. Soljacic, "High-contrast alloptical bistable switching in photonic crystal microcavities," Appl. Phys. Lett. 83, 2739-2741 (2003).

12. W. Chen and D. L. Mills, "Gap solitons and the nonlinear optical-response of superlattices," Phys. Rev. Lett. 58 160-163 (1987).

13. S. F. Mingaleev, Y. S. Kivshar, and R. A. Sammut, "Longrange interaction and nonlinear localized modes in photonic crystal waveguides," Phys. Rev. E 62, 5777-5782 (2000).

14. S. Mookherjea and A. Yariv, "Kerr-stabilized superresonant modes in coupled-resonator optical waveguides," Phys. Rev. E 66, 046610 (2002).

15. S. Pereira, P. Chak, and J. E. Sipe, "Gap-soliton switching in short microresonator structures," J. Opt. Soc. Am. B 19, 2191-2202 (2002).

16. P. Xie and Z. Q. Zhang, "Excitation of gap solitons, soliton trains, and soliton sets in finite-sized two-dimensional photonic crystals," Phys. Rev. E 69, 036601 (2004).

17. A. R. McGurn and G. Birkok, "Transmission anomalies in Kerr media photonic crystal circuits: intrinsic localized modes," Phys. Rev. B 69, 235105 (2004).

18. P. Bienstman and R. Baets, "Optical modelling of photonic crystals and VCSELs using eigenmode expansion and perfectly matched layers," Opt. Quantum Electron. 33, 327-341 (2001).

19. CAMFR simulation software is freely available from http:// camfr.sourceforge.net/.

20. K. C. Huang, P. Bienstman, J. D. Joannopoulos, K. A. Nelson, and S. Fan, "Phonon-polariton excitations in photonic crystals,” Phys. Rev. B 68, 075209 (2003). 
21. D. Pissoort, B. Denecker, P. Bienstman, F. Olyslager, and D. De Zutter, "Comparative study of three methods for the simulation of two-dimensional photonic crystals," J. Opt. Soc. Am. A 21, 2186-2195 (2004).

22. B. Maes, P. Bienstman, and R. Baets, "Modeling of Kerr nonlinear photonic components with mode expansion," Opt. Quantum Electron. 36, 15-24 (2004).

23. M. Soljacic, C. Luo, J. D. Joannopoulos, and S. Fan,
"Nonlinear photonic crystal microdevices for optical integration," Opt. Lett. 28, 637-639 (2003).

24. P. Chak, J. E. Sipe, and S. Pereira, "Lorentzian model for nonlinear switching in a microresonator structure," Opt. Commun. 213, 163-171 (2002).

25. J. E. Sipe, L. Poladian, and C. M. de Sterke, "Propagation through nonuniform grating structures," J. Opt. Soc. Am. A 11, 1307-1320 (1994). 